\title{
Behaviour and modelling of aluminium alloy AA6060 subjected to a wide range of strain rates and temperatures
}

\author{
Vincent Vilamosa, Tore Børvik, Odd Sture Hopperstad, and Arild Holm Clausen ${ }^{\mathrm{a}}$ \\ Structural Impact Laboratory (SIMLab), Department of Structural Engineering, Norwegian University of Science and \\ Technology (NTNU), Trondheim, Norway
}

\begin{abstract}
The thermo-mechanical behaviour in tension of an as-cast and homogenized AA6060 alloy was investigated at a wide range of strains (the entire deformation process up to fracture), strain rates $\left(0.01-750 \mathrm{~s}^{-1}\right)$ and temperatures $\left(20-350{ }^{\circ} \mathrm{C}\right)$. The tests at strain rates up to $1 \mathrm{~s}^{-1}$ were performed in a universal testing machine, while a split-Hopkinson tension bar (SHTB) system was used for strain rates from 350 to $750 \mathrm{~s}^{-1}$. The samples were heated with an induction-based heating system. A typical feature of aluminium alloys at high temperatures is that necking occurs at a rather early stage of the deformation process. In order to determine the true stress-strain curve also after the onset of necking, all tests were instrumented with a digital camera. The experimental tests reveal that the AA6060 material has negligible strain-rate sensitivity (SRS) for temperatures lower than $200^{\circ} \mathrm{C}$, while both yielding and work hardening exhibit a strong positive SRS at higher temperatures. The coupled strain-rate and temperature sensitivity is challenging to capture with most existing constitutive models. The paper presents an outline of a new semi-physical model that expresses the flow stress in terms of plastic strain, plastic strain rate and temperature. The parameters of the model were determined from the tests, and the stress-strain curves from the tests were compared with the predictions of the model. Good agreement was obtained over the entire strain rate and temperature range.
\end{abstract}

\section{Introduction}

Aluminium alloys in the AA6xxx series are often used for extruded profiles and rolled sheets or plates. Such components occur frequently in fields of engineering where lightweight designs are required, for instance safety parts in vehicles and different protective structures. Such parts have to resist rapid loading. High strain rate occurs often in combination with elevated temperatures in impact situations, but also in metal forming operations like extrusion and rolling. Common for all these fields of application is that numerical tools and the finite element method are important in the design process. Accurate predictions require material models that represent the physical response in an adequate way.

There are very few systematic experimental studies of coupled effects between strain rate and temperature in the literature. A particular feature associated with tension tests at high temperatures is that necking occurs at a comparatively small deformation, calling for local measurements of the strains in the neck in order to determine the true stress-strain curve.

This paper presents results from uniaxial tension tests on an AA6060 alloy at a wide range of strain rates and temperatures. The test data are fitted to a recently proposed constitutive model that provides a close representation of the observed behaviour. A more comprehensive presentation of the dynamic test rig allowing for different temperatures, the uniaxial test results and the material model is provided in three articles by Vilamosa et al. [1-3].

\footnotetext{
${ }^{a}$ Corresponding author: arild.clausen@ntnu.no
}

\section{Materials and methods}

This investigation involves the two aluminium alloys Al$0.5 \mathrm{Mg}-0.45 \mathrm{Si}$ and $\mathrm{Al}-0.45 \mathrm{Mg}-0.4 \mathrm{Si}$. Both are within the window of the AA6060 alloy. Their mechanical response is similar [2], and they are therefore treated in common in the numerical part of this paper.

The material was delivered as cast and homo-genised billets by Hydro Aluminium, and was naturally aged for about 18 months prior to testing. The sample shown in Fig. 1 was applied in all tests.

The thermo-mechanical test series consisted of tension tests at different temperatures $\left(20^{\circ} \mathrm{C}, 200^{\circ} \mathrm{C}, 250^{\circ} \mathrm{C}\right.$, $300^{\circ} \mathrm{C}$ and $\left.350{ }^{\circ} \mathrm{C}\right)$ and nominal strain rates $\left(0.01 \mathrm{~s}^{-1}\right.$, $1 \mathrm{~s}^{-1}, 350 \mathrm{~s}^{-1}$ and $\left.750 \mathrm{~s}^{-1}\right)$. The tests at low to moderate strain rates were carried out in a universal testing machine, while a SHTB system was employed for the dynamic tests. In advance, it was checked that the materials are isotropic [2].

\subsection{Thermo-mechanical tension tests}

\subsubsection{Quasi-static tests}

The tests at nominal strain rates $\dot{e}$ of $0.01 \mathrm{~s}^{-1}$ and $1 \mathrm{~s}^{-1}$ were carried out under displacement control in a Zwick-Roell testing machine, applying a cross-head velocity of $0.05 \mathrm{~mm} / \mathrm{s}$ and $5 \mathrm{~mm} / \mathrm{s}$. A water-cooled induction heating system delivered by MSI Automation was employed to heat the samples. The heating rate was about $10^{\circ} \mathrm{C} / \mathrm{s}$. The temperature was kept stable during the test with a feed-back loop provided by the temperature measurement system (a laser-based pyrometer delivered 


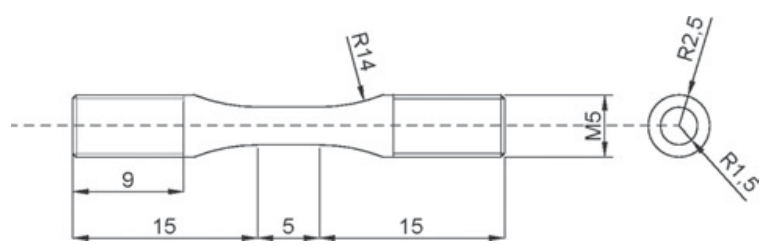

Figure 1. Tensile test specimen. Measures in $\mathrm{mm}$.

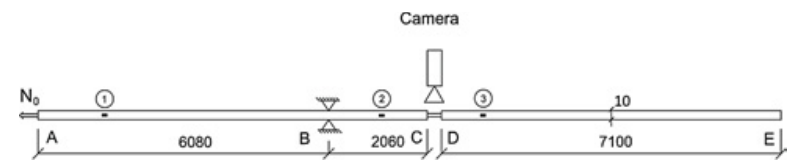

Figure 2. Sketch of SHTB system. Measures in mm.

by LumaSense Technologies). Vilamosa et al. [2] provide more information on this experimental set-up.

It was pointed out in Sect. 1 that necking occurs at an early stage of deformation at elevated temperatures. Therefore, it was required to determine the local strain inside the neck The deformation of the samples was captured by a digital camera (Prosilica GC2450) having a 5 megapixel Sony ICX625 CCD sensor. Frames were recorded until fracture with a sampling rate of $2 \mathrm{~Hz}$ $\left(\dot{e}=0.01 \mathrm{~s}^{-1}\right)$ and $15 \mathrm{~Hz}\left(\dot{e}=1 \mathrm{~s}^{-1}\right)$. The pixel size was determined before each test by measuring the initial diameter $D_{0}$ of the samples both with the camera and a digital calliper. After the tests, an edge detection script was applied to determine the minimum cross section diameter $D_{s}$ of the sample [1]. Assuming constant volume during plastic deformation, the local logarithmic strain $\varepsilon$ was determined from

$$
\varepsilon=\ln \left(\frac{A_{0}}{A_{s}}\right)=2 \ln \left(\frac{D_{0}}{D_{s}}\right)
$$

where $A_{0}$ is the initial area of the sample's cross-section and $A_{s}=(\pi / 4) D_{s}^{2}$ is the minimum cross-section area during testing. The true stress $\sigma$ was found by dividing the force $F$ measured by the load cell with the current area, viz.

$$
\sigma=\frac{F}{A_{s}} .
$$

Finally, the plastic strain $\varepsilon^{p}$ was determined from the following relation

$$
\varepsilon^{p}=\varepsilon-\frac{\sigma}{E}
$$

where $E$ is Young's modulus at room temperature.

\subsubsection{Dynamic tests}

A split-Hopkinson tension bar (SHTB) system, see Fig. 2, was used in the dynamic tests at approx. $\dot{e}=350 \mathrm{~s}^{-1}$ and $\dot{e}=750 \mathrm{~s}^{-1}$. It consists of an $8140 \mathrm{~mm}$ long input bar $(\mathrm{ABC})$ and a $7100 \mathrm{~mm}$ long transmission bar (DE). The sample is located between points $C$ and $D$. Both bars have diameter $10 \mathrm{~mm}$, and are made of high-strength steel with Young's modulus $210 \mathrm{GPa}$ at room temperature.

The bars are instrumented with three strain gauge stations at the locations (1), (2) and (3). Strain gauge (1) is used to monitor the level of the pre-tension force $N_{0}$. The stress wave propagation during the test is measured with the gauges at (2) and (3). Applying the classical Kolsky equations, the data from these gauges can be used to determine the nominal stress, strain and strain rate in the specimen. Provided that the deformation of the sample is homogeneous, i.e., before the onset of necking, it is straightforward to calculate the true stress and logarithmic strain from the nominal data.

Also the dynamic tests had to be instrumented with a camera in order to find $\sigma$ and $\varepsilon$ in the comparatively large phase of the test after necking. A SA1.1 Photron highspeed camera with frame rate $100 \mathrm{kHz}$ was applied for this purpose. The minimum diameter of the sample during the test was found from the digital pictures in the same way as in the quasi-static tests. Equation (1) was thereafter applied to find the logarithmic strain. The force in the sample was determined by use of the conventional method for analysis of SHTB tests. It has been shown by Chen et al. [4] that there is no dispersion in the SHTB system shown in Fig. 2. The force $F$ in the specimen is therefore proportional with the strain $\varepsilon_{T}$ measured at position (3) of the transmission bar, i.e.

$$
F=A_{b} E_{b} \varepsilon_{T}
$$

where $A_{b}$ and $E_{b}$ respectively are the cross-section area and Young's modulus of the bar. Subsequently, the true stress was found with Eq. (2), and Eq. (3) gives the plastic strain.

The induction heating equipment presented in Sect. 2.1.1 was used also in the dynamic tests. It was demonstrated by Vilamosa et al. [1] that the heated sample did not disturb the wave propagation in the bars. Further, the camera-based measurement technique was validated against the conventional approach (Kolsky equations) for strain determination before necking. A more comprehensive presentation of the experimental set-up for the thermo-mechanical tests is provided by Vilamosa et al. $[1,2]$.

\subsection{Data processing}

All samples exhibited pronounced necking that increased with increasing temperature. This results in a threedimensional stress state in the neck. This is handled with the Bridgman relation, which takes the shape of the neck and the diameter of the smallest cross-section into account. Such information is available from the digital pictures. The equivalent stress $\sigma_{e q}$ is determined from

$$
\sigma_{e q}=\frac{\sigma}{\left(1+\frac{4 R}{D_{s}}\right) \ln \left(1+\frac{D_{s}}{4 R}\right)}
$$

where $R$ is the mean radius of the curvature of the neck. This radius was estimated from each frame with a least squares method [1].

Small oscillations were observed in the equivalent stress - plastic strain curves from all tests, in particular the dynamic ones from SHTB. As the subsequent analysis requires values of $\sigma_{e q}$ at certain levels of plastic strain $\varepsilon^{p}$, see Sect. 4.2, these oscillations should be removed. For this 


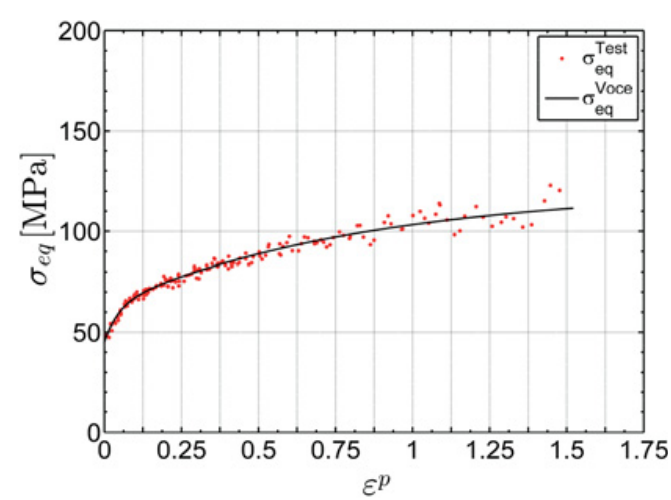

Figure 3. Equivalent stress - plastic strain curves determined from a test (discrete data points) and fitted to Eq. (6) for a representative test at a nominal strain rate at approx. $350 \mathrm{~s}^{-1}$ and temperature of $350{ }^{\circ} \mathrm{C}$.

purpose, all stress-strain curves were fitted to a two-term Voce relation extended with a linear hardening term

$$
\sigma_{e q}=\sigma_{0}+\sum_{i=1}^{2} Q_{i}\left(1-\exp \left(-\frac{\theta_{i}}{Q_{i}} \varepsilon^{p}\right)\right)+H \varepsilon^{p}
$$

The five parameters $Q_{i}, \theta_{i}$ and $H$ were fitted to the part of the $\sigma_{e q}-\varepsilon^{p}$ curve with plastic strain larger than 0.01, thereby omitting the initial part of the curve where there is a lack of equilibrium in the SHTB tests. Considering a representative dynamic test, Fig. 3 shows the $\sigma_{e q}-\varepsilon^{p}$ curves as obtained in the test and as fitted with Eq. (6).

In all tests, the local plastic strain rate $\dot{\varepsilon}^{p}$ in the necked section was obtained by numerical differentiation of the plastic strain vs. time curve.

\section{Experimental results}

Equivalent stress - plastic strain curves from all tests are shown in Fig. 4. The solid and dotted lines refer to the two slightly different AA6060 alloys, respectively Al- $0.5 \mathrm{Mg}$ $0.45 \mathrm{Si}$ and $\mathrm{Al}-0.45 \mathrm{Mg}-0.4 \mathrm{Si}$.

Figure 4 reveals that 1 or 2 samples of each alloy were tested at each combination of strain rate and temperature. The scatter between replicate tests was small. It appears that the response of the two alloys is almost identical. They are therefore treated in common in the subsequent data analysis. Some curves are clipped at the onset of necking. In these cases, the neck was not visible in the digital photos because it was hidden behind the coil of the induction heater.

Figure 4 shows that the material exhibits a decrease in both yield strength and work hardening with increasing temperature for the four levels of nominal strain rate. It is interesting to notice that the curves at different rates are rather similar at room temperature, while the work hardening increases considerably with $\dot{e}$ at $350{ }^{\circ} \mathrm{C}$. This interaction effect between strain rate and temperature is in general not well captured by existing constitutive models for use in finite element (FE) simulations [2].
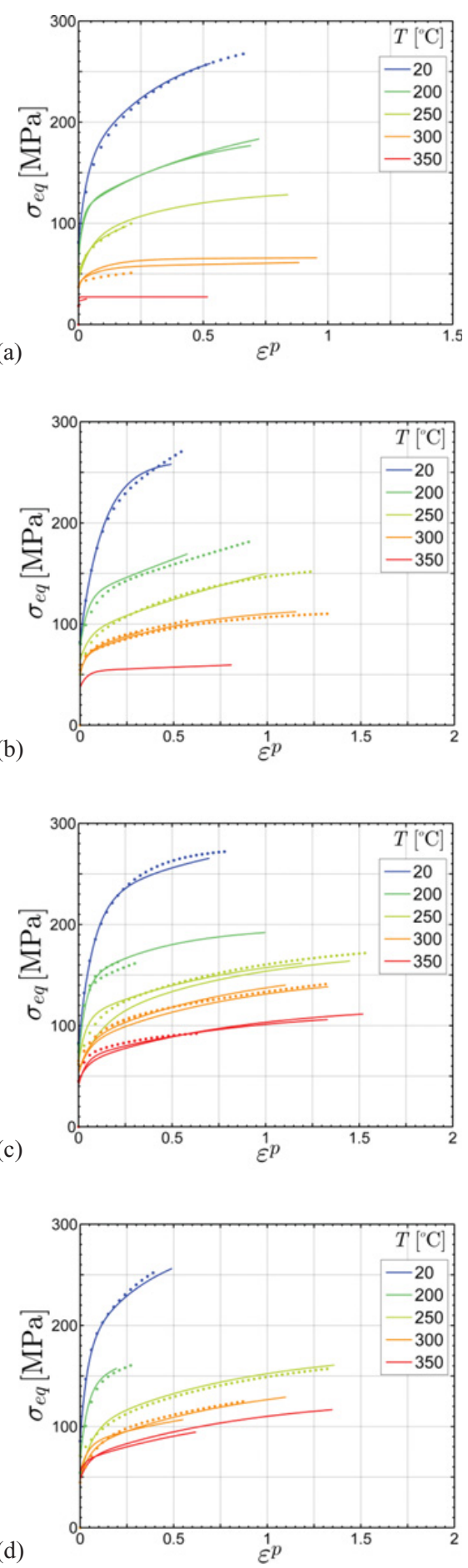

Figure 4. Equivalent stress - plastic strain curves for Al-0.5Mg$0.45 \mathrm{Si}$ (solid lines) and $\mathrm{Al}-0.45 \mathrm{Mg}-0.4 \mathrm{Si}$ (dotted lines) at all temperatures and nominal strain rate levels of (a) $0.01 \mathrm{~s}^{-1}$, (b) $1 \mathrm{~s}^{-1}$, (c) $350 \mathrm{~s}^{-1}$ and (d) $750 \mathrm{~s}^{-1}$.

\section{Constitutive model}

The most employed constitutive models in FE codes are of phenomenological nature. Such models are empirical, i.e., based on experimental observations, they have few parameters, and are often expressed as algebraic equations that are easy to implement in the code. The physically based models provide a different approach. They seek to 
relate the flow stress to the evolution of dislocation density and the thermal activation energies that are required for dislocations to overcome obstacles.

As an attempt to capture the response of AA6060, see Fig. 4, in FE simulations, a thermoelastic-thermoviscoplastic model has been developed. It assumes corotational hypoelastic plasticity, implying that the elastic strains should be small. Since the considered material is isotropic, the model incorporates isotropic tensors for the thermo-elastic moduli and also the isotropic Hershey yield function. Vilamosa et al. [3] provides a more comprehensive presentation of the general framework of the model. The next section will, however, outline the main ideas of how the flow stress is modelled.

\subsection{Flow stress and work hardening}

Bergström [5] suggested to split the flow stress $\sigma_{f}$ into three terms

$$
\sigma_{f}(R, \dot{\bar{\varepsilon}}, T)=\sigma_{a}(T)+\sigma_{v}(\dot{\bar{\varepsilon}}, T)+R .
$$

The athermal yield stress $\sigma_{a}(T)$ is dependent on temperature only; not strain rate. It is assumed that the temperature sensitivity of $\sigma_{a}$ is the same as for the shear modulus (and Young's modulus) [3]. This stress captures the strengthening effect from particles.

The instantaneous viscous stress $\sigma_{v}(\dot{\bar{\varepsilon}}, T)$ depends on both strain rate and temperature. Thus, a change of either of these variables, or both, will cause a change of $\sigma_{v}$. As outlined by Vilamosa et al. [3], the expression for $\sigma_{v}$ combines an Arrhenius type of expression with an activation energy profile and also a regularization to avoid instabilities at low strain rates. The viscous stress represents the presence of obstacles.

The work hardening $R$ is related to the dislocation density $\rho$, i.e., $R$ increases with $\rho$. It depends indirect-ly on strain rate and temperature through the evolution rule for $\rho$, and in addition on temperature through the shear modulus [3]. The final expression for the evolution of work hardening as function of plastic strain reads

$$
\frac{d R}{d \varepsilon^{p}}=\theta_{0} \exp \left(-\frac{R}{R_{s}}\right)
$$

where $\theta_{0}$ is a temperature sensitive term representing the initial slope of the work hardening and $R_{S}$ is a saturation value which depends both on temperature and strain rate. At the strain rates covered in this study, the dislocation density is believed to depend mainly on dynamic recovery which is a thermally activated process. The significant strengthening observed in the dynamic tests, see Fig. 4(c) and (d), is attributed to a change of the strain rate sensitivity of the material resulting in a reduced dynamic recovery $[3,6]$. This is implemented in the model. The new constitutive model might therefore be applied for this class of materials up to relatively high strain rates of order $10^{4} \mathrm{~s}^{-1}$.

\subsection{Evaluation of the model}

As the two AA6060 alloys exhibit similar response at $350 \mathrm{~s}^{-1}$ and $750 \mathrm{~s}^{-1}$, the constitutive model is calibrated with the test data obtained at nominal strain rates of

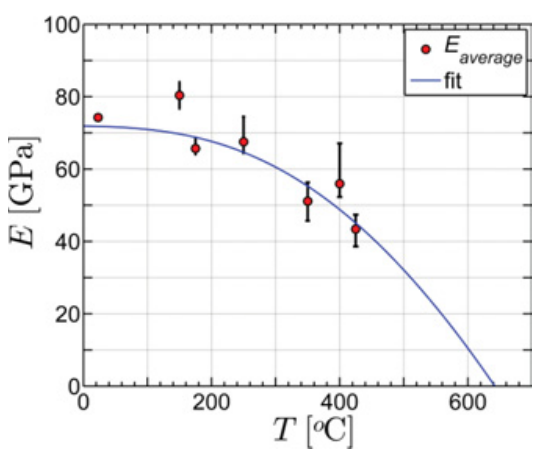

Figure 5. Temperature sensitivity of Young's modulus. The bars denote the range of the measured values.
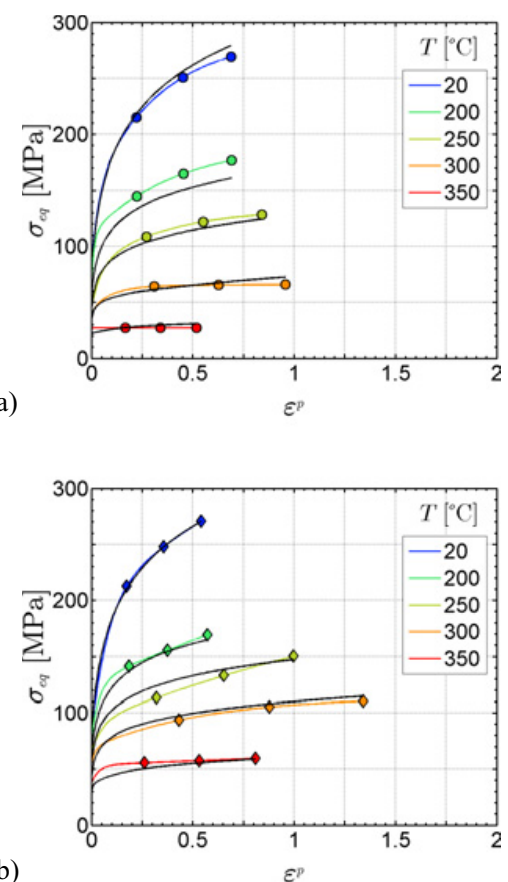

(b)

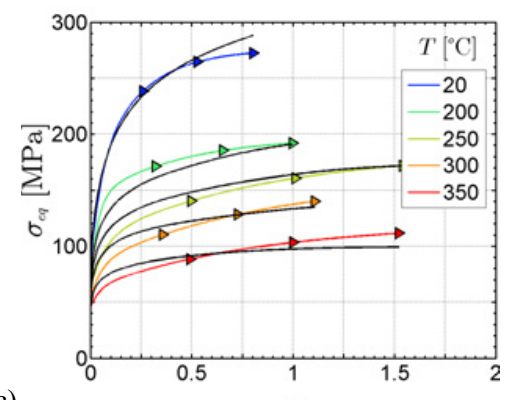

(c)

Figure 6. Comparison of equivalent stress - plastic strain curves from representative tests (lines with symbols) and the constitutive model (solid lines without symbols) at all temperatures and nominal strain rate levels of (a) $0.01 \mathrm{~s}^{-1}$, (b) $1 \mathrm{~s}^{-1}$ and (c) $350 \mathrm{~s}^{-1}$.

$0.01 \mathrm{~s}^{-1}, 1 \mathrm{~s}^{-1}$ and $350 \mathrm{~s}^{-1}$. In addition, information about the temperature sensitivity of Young's modulus is required, see Fig. 5. Vilamosa et al. [3] provide a more comprehensive outline of the parameter identification procedure.

Figure 6 compares results from representative tests (taken from Fig. 4) with equivalent stress - plastic strain 
(a)

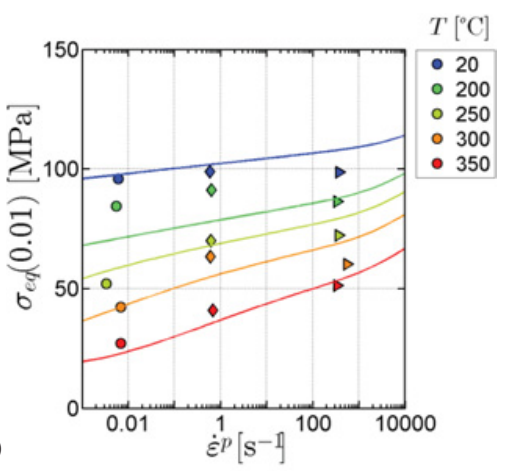

(b)

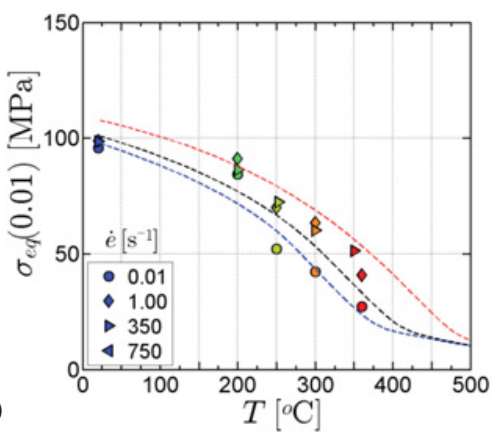

Figure 7. Comparison of equivalent stress at a plastic strain of 0.01 in the tests (discrete points) and the model (lines) at (a) different strain rates, and (b) different temperatures.

curves predicted by the model. The entire spectre of strain rates and temperatures is covered in the figure. The agreement between the test data and the model is in general good at all levels of plastic strain. In particular, the model captures that the work hardening at the highest temperatures almost vanishes at $\dot{e}=0.01 \mathrm{~s}^{-1}$, while it is significantly larger at $\dot{e}=350 \mathrm{~s}^{-1}$.

A closer look reveals, however, that the model underestimates the yield strength in some cases, in particular at temperatures around $200{ }^{\circ} \mathrm{C}$. Such a mismatch has also consequences for the prediction of the flow stress. On the other hand, the flow stress is overestimated at high temperatures under dynamic condi-tions at low plastic strains.

An alternative way to compare the results is to extract the equivalent stress at certain levels of plastic strain. The values of $\varepsilon^{p}=0.01, \varepsilon^{p}=0.05$ and $\varepsilon^{p}=0.6$ were chosen for this purpose because these strains represent different stages of work hardening. These three levels are addressed in Figs. 7, 8 and 9, respectively, where sub-figures (a) address the response as function of plastic strain rate, while sub-figures (b) have temperature at the abscissa axis. The three dashed lines in sub-figures (b) refer to strain rates of $0.01 \mathrm{~s}^{-1}, 1 \mathrm{~s}^{-1}$ and $350 \mathrm{~s}^{-1}$.

At high strain rates, there is a significant adiabatic heating during a tension test. The associated increase of temperature $\Delta T$ is estimated by

$$
\Delta T=\int_{0}^{\varepsilon_{p}} \chi \frac{\sigma_{e q} d \varepsilon_{p}}{\rho C_{p}}
$$
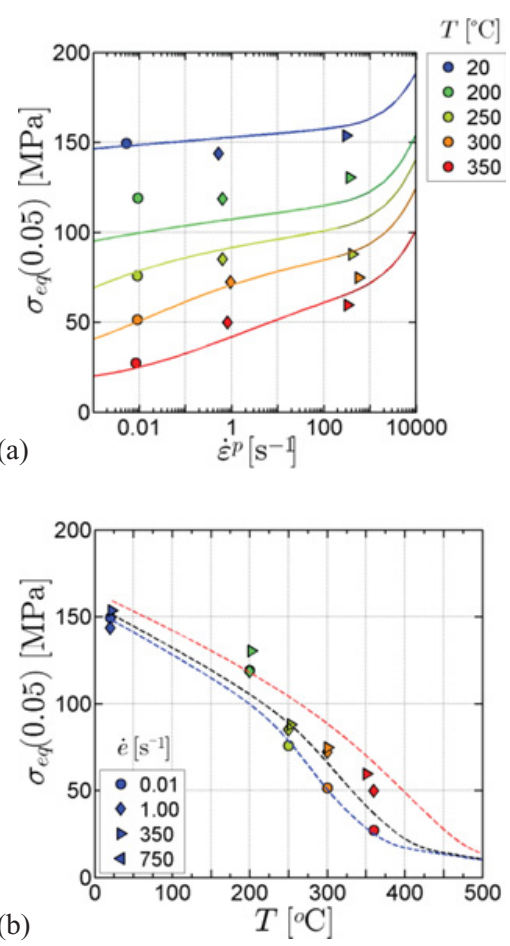

Figure 8. Comparison of equivalent stress at a plastic strain of 0.05 in the tests (discrete points) and the model (lines) at (a) different strain rates, and (b) different temperatures.

(a)

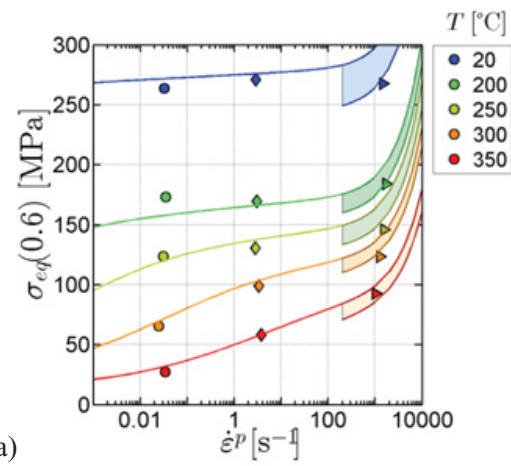

(b)

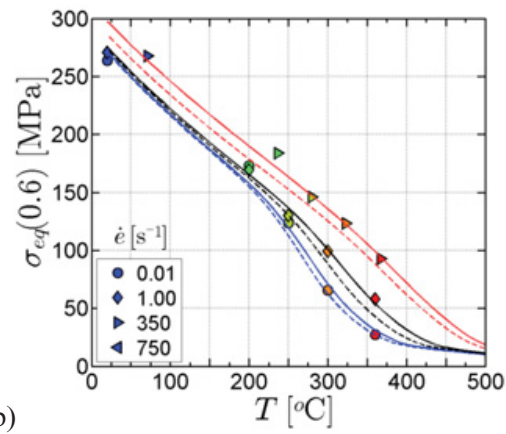

Figure 9. Comparison of equivalent stress at a plastic strain of 0.6 in the tests (discrete points) and the model (lines) at (a) different strain rates, and (b) different temperatures. The shaded areas in (a) address the difference between adiabatic and iso-thermal conditions. The solid lines in (b) represent the increase of strain rate at the neck. 
where $\rho$ is the density, $C_{p}$ is the specific heat capacity, and $\chi$ is the Taylor-Quinney coefficient. Both $C_{p}$ and $\chi$ are assumed to be temperature independent. The temperature increase gives a softening effect on $\sigma_{e q}$, in particular at $\varepsilon^{p}=0.6$. The effect of $\Delta T$ is addressed through the shaded area in Fig. 9(a).

Sub-figures (a) in Figs. 7-9 show clearly that the material is close to rate insensitive at room temperature, while $\sigma_{e q}$ increases significantly with rate at the high temperatures. This tendency is present around the onset of yielding (Fig. 7) as well as after considerable work hardening (Fig. 9). The model captures these observations. Sub-figures (b) show that the softening with increasing temperature is more prominent at quasi-static loading conditions than at high strain rates. The model represents also this feature rather accurately.

In addition to a more comprehensive derivation of the model, Vilamosa et al. [3] also compares the predict-tions of the model with experimental results for an AA6082 alloy. This material has a higher content of the alloying elements Mg and Si than AA6060. Also here, the model gives a close reconstruction of the stress-strain curves. Moreover, finite element simulations of both the quasistatic and dynamic tension tests, in the latter case including the entire SHTB set-up, show that the model is capable of describing the behaviour at different combinations of strain rate and temperature.

\section{Conclusion}

This paper presented a series of thermo-mechanical tension tests on two slightly different AA6060 alloys. The tests were performed at strain rates between $0.01 \mathrm{~s}^{-1}$ and $750 \mathrm{~s}^{-1}$ and at temperatures between $20^{\circ} \mathrm{C}$ and $350{ }^{\circ} \mathrm{C}$. A universal testing machine was used at low to medium strain rates, while a split-Hopkinson tension bar system was employed at the dynamic strain rates. The samples were heated with an induction apparatus. A camera-based system was used to determine the local strains also after the onset of necking.

A strong coupling between strain rate and temperature sensitivity was found in the tests. The material is close to insensitive to strain rate at room temperature. At the high temperatures, however, there is a significant increase of the yield stress and work hardening with strain rate.

The paper also outlined the main features of a new constitutive model. The model gave in general a faithful representation of the experimental observations.

The authors would like to express gratitude to M.T. Auestad at SIMLab, NTNU, for his assistance with the experimental work. The contributions from PhD E. Fagerholt at SIMLab, NTNU, PhD S.R. Skjervold at SAPA and Professor B. Holmedal at Department of Materials Science and Engineering, NTNU, are also acknowledged.

\section{References}

[1] V. Vilamosa, A.H. Clausen, E. Fagerholt, O.S. Hopperstad, T. Børvik, Strain 50, 223-235 (2014)

[2] V. Vilamosa, A.H. Clausen, T. Børvik, S.R. Skjervold, O.S. Hopperstad, Submitted for journal publication (2015)

[3] V. Vilamosa, A.H. Clausen, T. Børvik, B. Holmedal, O.S. Hopperstad, Submitted for journal publication (2015)

[4] Y. Chen, A.H. Clausen, O.S. Hopperstad, M. Langseth, Int. J. Impact. Eng. 38, 824-836 (2011)

[5] Y. Bergström, Mat Sci Eng. 5, 193-200 (1970)

[6] P.S. Follansbee, U.F. Kocks, Acta Metall. 36, 81-93 (1988) 